\title{
Opposing Oxytocin Effects on Intergroup Cooperative Behavior in Intuitive and Reflective Minds
}

\author{
Yina Ma*,1,2, Yi Liu ${ }^{2,3}$, David G Rand ${ }^{4}$, Todd F Heatherton ${ }^{5}$ and Shihui Han*,2,3 \\ 'Lieber Institute for Brain Development, Johns Hopkins University School of Medicine, Baltimore, MD, USA; ${ }^{2}$ Department of Psychology, \\ Peking University, Beijing, China; ${ }^{3}$ PKU-IDG/McGovern Institute for Brain Research, Peking University, Beijing, China; ${ }^{4}$ Department of Psychology, \\ Yale University, New Haven, CT, USA; ${ }^{5}$ Psychological and Brain Sciences, Dartmouth College, Hanover, NH, USA
}

\begin{abstract}
People often favor ingroup over outgroup members when choosing to cooperate. Such ingroup-favored cooperation is promoted by oxytocin - a neuropeptide shown to facilitate social cognition and that has emerged as a pharmacological target for treatments of social functioning deficits. The current study applied a dual-process model to investigate whether and how intuitive and reflective cognitive styles affect the oxytocin-motivated ingroup favoritism in cooperation. We examined oxytocin effects on ingroup favoritism in a double-blind, placebo-controlled between-subjects design where cognitive processing (intuition vs reflection) was experimentally manipulated in healthy Chinese males $(n=150)$. We also supplemented this experimental manipulation with an individual difference analysis by assessing participants' inclination toward intuition or reflection in daily life. Intranasal administration of oxytocin (vs placebo) increased ingroup favoritism among participants primed to be intuitive or those who preferred intuition in daily life. In contrast, oxytocin decreased ingroup favoritism in participants primed to rely on reflective thinking or those who preferred reflective decision-making in daily life. Our results demonstrate that oxytocin has distinct functional roles when different cognitive styles (ie, intuition vs reflection) are promoted during social cooperation in a group situation. Our findings have implications for oxytocin pharmacotherapy of social dysfunction in that whether the effects of oxytocin on social functioning are facilitative, debilitative, or null, depends on an individual's cognitive style.

Neuropsychopharmacology (20I5) 40, 2379-2387; doi:I0.I038/npp.20I5.87; published online I5 April 2015
\end{abstract}

\section{INTRODUCTION}

Social cooperation is indispensable in human societies (Nowak and Sigmund, 2005; Rand and Nowak, 2013). However, people do not always cooperate equally with others. We often favor members of our own group over those from other groups (ie, ingroup favoritism; Tajfel et al, 1971; Arrow, 2007). Recent research has revealed that oxytocin, a neuropeptide that facilitates social affiliation and social cognition (Kosfeld et al, 2005; Bartz and Hollander, 2006; Bartz et al, 2010, 2011a), motivates ingroup favoritism in social behavior (De Dreu, 2012; van IJzendoorn and Bakermans-Kranenburg, 2012). Intranasal administration of oxytocin (compared with placebo) enhanced ingroup favoritism in social trust (van IJzendoorn and BakermansKranenburg, 2012), empathy for pain (Sheng et al, 2013), and cooperation (De Dreu et al, 2010, 2011; De Dreu, 2012). What remains unknown is whether and how the oxytocinmotivated ingroup favoritism is modulated by individuals'

\footnotetext{
*Correspondence: Dr Y Ma, Lieber Institute for Brain Development, Johns Hopkins University School of Medicine, 855 North Wolfe Street, Suite 300, 3rd Floor, Baltimore, MD 21205, USA, Tel: + I 603443 2062, E-mail: yinama0 I@gmail.com or Dr S Han, Department of Psychology, Peking University, 52 Haidian Street, Beijing 100080, China, Tel: +1 86106275 9138, Fax: + 86106276 1081, E-mail: shan@pku.edu.cn Received 3 November 2014; revised 16 March 2015; accepted 17 March 20I5; accepted article preview online 25 March 2015
}

cognitive styles. Unraveling whether and how one's cognitive style influences the oxytocin effect helps to understand how the biological pathways activated by oxytocin interact with different cognitive processes, to better predict oxytocin effects on social cognition across individuals, and to provide cognitive mechanisms to explain the inconsistence of oxytocin effects in the literature (Bartz et al, 2011a). To address this issue also has important implications for oxytocin-based clinical practice.

Oxytocin, known for its anxiolytic action and positive effects on social cognition (Bartz and Hollander, 2006; Heinrichs et al, 2009; Meyer-Lindenberg et al, 2011), is emerging as a pharmacological target for novel treatments of mental disorders, especially those with social dysfunction (Heinrichs et al, 2009; Insel, 2010; Bakermans-Kranenburg and van IJzendoorn, 2013), such as autism spectrum disorder, social anxiety disorder, and schizophrenia. However, the oxytocin effects observed in healthy or patient populations are often weak and inconsistent, even showing opposite patterns in the literature (Bartz et al, 2011a, b; Bakermans-Kranenburg and van IJzendoorn, 2013). Oxytocin effects are sensitive to contexts and individual differences (Bartz et al, 2011a, b), and patients with social functioning deficits are characterized by different cognitive processing styles (Kashdan and Hofmann, 2008; Binelli et al, 2014). Therefore, understanding how cognitive styles influence oxytocin effects on social functioning is critical for successful clinical translation to be achieved. 
The current study applied a dual-process model to investigate whether and how one's cognitive style affected the oxytocinmotivated ingroup favoritism in cooperation. The dual-process model proposes two distinct cognitive systems to produce decisions. Individuals with intuitive cognitive style prefer frugal, heuristic, and fast responses, whereas reflective individuals favor deliberative, analytic, and slow responses (Kahneman, 2011; Evans, 2008). It has been shown that intuitive and reflective cognitive styles play opposing roles in cooperative decision-making (Rand et al, 2012) such that intuition tends to support cooperation while reflection favors selfishness (Rand et al, 2012; Zaki and Mitchell, 2013). Neuroscience research has documented that intuition is supported mainly by the limbic system, including the amygdala, striatum, midbrain, nucleus accumbens (NAcc), ventral medial prefrontal cortex (mPFC), and orbitofrontal (OFC; Lieberman, 2007; Dalgleish, 2004). In contrast, reflection is supported by the lateral prefrontal cortex, dorsal anterior cingulate cortex, medial temporal lobe, and posterior parietal cortex (Lieberman, 2007; Miller and Cohen, 2001). Oxytocin is synthesized in the hypothalamus and projects from the hypothalamus to the amygdala, striatum, suprachiasmatic nucleus, and brainstem (Ludwig and Leng, 2006; Donaldson and Young, 2008). Intranasal administration of oxytocin has been shown to mainly modulate neural activity in the amygdala (Domes et al, 2007; Petrovic et al, 2008; Baumgartner et al, 2008), midbrain/striatum/NAcc (Baumgartner et al, 2008; Gordon et al, 2013; Groppe et al, 2013), mPFC (Petrovic et al, 2008; Gordon et al, 2013), and OFC (Petrovic et al, 2008; Gordon et al, 2013). These findings suggested that the oxytocinergic system and the intuition system involved common neural underpinnings. In addition, behavioral studies have shown evidence for distinct oxytocin effects on fast and slow emotion recognition. Oxytocin facilitated recognition of happy expression during fast exposure but enhanced recognition of fearful expression during slow recognition (Shahrestani et al, 2013).

These behavioral and neuroscience findings allow us to hypothesize that oxytocin promotes ingroup favoritism when intuition is favored. Reflective deliberation, however, might overrule the effect of oxytocin or even reverse it. We tested this hypothesis in a double-blind, placebo-controlled betweensubjects design by combining intranasal administration of oxytocin and cognitive-style manipulation. Two complementary approaches were adopted to test our hypothesis. First, we assessed whether conceptual priming that temporarily promoted intuition or reflection affected the oxytocin effects on ingroup favoritism during a public goods game (PGG) with ingroup or outgroup members. Second, given that people differ in the inclination toward intuition or reflection in daily life (Evans, 2008; Lieberman, 2007), we examined whether the oxytocin effect on ingroup favoritism during PGG differed between individuals who preferred intuition or reflection in daily-life decision-making.

\section{MATERIALS AND METHODS}

\section{Participants}

This study recruited 150 male Chinese college students as paid volunteers. Data analyses consisted of 38 participants in the intuition priming under placebo, 38 participants in the intuition priming under oxytocin, 37 participants in the reflection priming under placebo, and 37 participants in the reflection priming under oxytocin. The protocol was approved by a local ethics committee at the Department of Psychology, Peking University (Beijing, China). Each participant gave written informed consent before the study. All participants reported no history of neurological or psychiatric diagnoses. Exclusion criteria were any self-reported medical or psychiatric disorder, medication/drug/alcohol abuse. Participants were instructed to refrain from smoking or drinking (except water) for $2 \mathrm{~h}$ before the experiment. Previous studies reported gender differences in both oxytocin function (Macdonald, 2012; Fischer-Shofty et al, 2013; Rilling et al, 2014) and ingroup favoritism (van Vugt et al, 2007; Charness and Rustichini, 2011). In addition, studies examining oxytocin effects on social cognition and behaviors have been conducted mainly with men (van IJzendoorn and Bakermans-Kranenburg, 2012). Thus, the current study only recruited healthy males to avoid any potential confound of gender effects.

\section{Experimental Design and Analysis}

The current study used a 2 (Treatment: oxytocin $v s$ placebo) $\times 2$ (Cognitive styles: intuition $v s$ reflection $) \times 2$ (Group: ingroup vs outgroup) design. Cognitive styles here refer to intuition or reflection that is temporarily promoted by an experimental manipulation, or that participants reported using in daily-life decision-making. We defined ingroup favoritism as the differences in behavioral responses or self-reports (ie, contribution amount in PGG, rating of first impression, likeness) related to ingroup $v s$ outgroup members. We first examined Treatment $\times$ Cognitive-style interactions on ingroup favoritism during PGG. Comparison between oxytocin and placebo groups was then performed to examine the oxytocin effect separately for intuitive and reflective participants. Regression analyses were then performed to examine whether the Treatment $\times$ Cognitive-style interactions on ingroup favoritism during PGG continued to be reliable after controlling for age, education, ingroup/ outgroup game orders, comprehension, trait optimism, interpersonal trust scores, experience with similar experiment, trust rating of the experiment, and total contribution to ingroup and outgroup and ingroup favoritism in first impression, likeness.

\section{Experimental Procedure}

Participants were recruited in groups of 10 individuals (all were strangers to each other). They first completed the questionnaires, which measured current mood, trait optimism, interpersonal trust, and intuition/reflection importance in daily-life decision-making. Participants were then intranasally administered with oxytocin or placebo. After $35 \mathrm{~min}$, participants were invited to the cognitive-style priming procedure, economic games, and calculation task. Finally, participants filled out a survey for mood measurement and manipulation check (Supplementary Figure S1 for the experimental procedure).

Daily used cognitive style. We assessed participants' cognitive styles before administration of placebo or oxytocin. Participants were asked to answer two questions on a 10- 
point Likert scale $(1=$ not important at all; $10=$ extremely important): 'To what extent do you think your intuition/first instinct is important in daily-life decision-making?' and 'To what extent do you think reflection/careful reasoning is important in daily-life decision-making?' These two questions were randomly placed among another 18 unrelated questions to avoid potential influences of the two questions on their behaviors. We examined the oxytocin effect on ingroup favoritism during PGG separately for the intuition and reflection importance rating. Using median split on the rating scores of intuition importance, we categorized participants into 'intuition-important' (placebo: $n=39$; oxytocin: $n=37$ ) and 'intuition-unimportant' (placebo: $n=36$; oxytocin: $n=38$ ) groups, collapsing participants across different priming conditions. Median split on the rating scores of reflection importance categorized participants into 'reflection-important' (placebo: $n=39$; oxytocin: $n=38$ ) and 'reflection-unimportant' (placebo: $n=36$; oxytocin: $n=37$ ) groups.

Oxytocin administration. The procedure of oxytocin and placebo administration was similar to the previous work, that showed significant oxytocin effects on decision-making behaviors or ingroup favoritism (Kosfeld et al, 2005; De Dreu et al, 2010, 2011). A single intranasal dose of $24 \mathrm{IU}$ oxytocin or placebo (containing the active ingredients except for the neuropeptide) was self-administered by nasal spray about $35 \mathrm{~min}$ before the experimental task under experimenter supervision. The spray was administered to participants three times, and each administration consisted of one inhalation of $4 \mathrm{IU}$ into each nostril. In a double-blind, placebo-controlled, between-subjects design, 8 groups of 10 participants were randomly assigned to placebo administration and the other 8 groups of 10 participants to oxytocin administration. A group of 10 participants performing the experiment at the same time was assigned to the same treatment (oxytocin or placebo), so as to avoid potential influence of oxytocin to placebo between individuals.

Minimal group paradigm. The group relationship was temporarily induced after oxytocin or placebo administration by dividing the 10 participants into two groups, so as to build the ingroup and outgroup relationship (Supplementary Information, Section 1, and Supplementary Table S1 for group manipulation check). Participants were randomly assigned to one of two groups based on the color of cards they picked from a deck. 'Group Black' appears on the back of five cards and 'Group White' appears on the back of the other five cards. All participants were then provided with either a black or white $\mathrm{T}$-shirt according to which group they belonged to, so as to enhance group identity.

Cognitive-style manipulation. A cognitive process induction procedure was used to promote intuitive or reflective cognitive styles. The procedure was similar to that used in the previous studies (Rand et al, 2012; see Supplementary Information, Section 2.1 for details of cognitive-style manipulation and Section 2.2 for manipulation check). Before PGGs, participants were asked to write down a paragraph recalling an episode from their daily life that was consistent with the suggestion of intuition or reflection. We used a between-subjects design in which participants were randomly assigned to adopt a more intuitive or reflective cognitive style.

Public goods game. Participants played a pair of PGGs, once with three ingroup members and once with three outgroup members. We explicitly emphasized that players were randomly and anonymously grouped by computer and no one would be grouped with the same people twice so as to preserve the one-shot nature of the games. The order of ingroup and outgroup games was counterbalanced across participants. In each PGG, each player independently received $80 \mathrm{U}$ ( $10 \mathrm{U}$ are equal to 1 Chinese yuan). Players decided how much money to contribute to a common project $v s$ how much to keep for themselves. All contributions would be doubled and split four ways. After reading the instructions on a computer screen, participants were informed about the group identity of the other three players. They then entered their contribution amounts. After the two decisions were made, participants were asked to predict the average contribution of the other three ingroup and outgroup members, respectively. To incentivize this prediction, we offered an extra monetary reward of $20 \mathrm{U}$ to participants depending on the accuracy of their prediction. At the end of the PGG, participants answered two comprehension questions to check whether they understood the payoff structure. Similar to previous studies (Rand et al, 2012, 2015), we controlled for comprehension by including comprehension as a covariate in the analysis. We included comprehension questions after the contribution decision to avoid the possibility of pushing participants into a reflective mindset before their decision-making.

\section{RESULTS}

\section{Distinct Oxytocin Effects on Ingroup Favoritism in Intuition vs Reflection Mindsets}

Ingroup favoritism was defined as the contribution to ingroup minus that to outgroup during PGGs. A 2 (Treatment: oxytocin $v s$ placebo) $\times 2$ (Priming: intuition $v s$ reflection) univariate analysis of variance (ANOVA) of ingroup favoritism revealed a significant interaction of Treatment $\times$ Priming on ingroup favoritism $(F(1,146)$ $=10.099, p=0.002, \eta^{2}=0.065$; Figure 1a), indicating discrepant oxytocin effects on ingroup favoritism in participants primed to favor intuition vs reflection. Specifically, oxytocin significantly enhanced ingroup favoritism when intuition was encouraged $(\mathrm{F}(1,74)=7.163, p=0.009$, $\eta^{2}=0.088$, whereas oxytocin significantly decreased ingroup favoritism when reflection was favored $(\mathrm{F}(1,72)=4.040$, $p=0.048, \eta^{2}=0.053$; Figure 1a; see Supplementary Figure S2 for individual contributions). The Treatment $\times$ Priming interaction on ingroup favoritism remained significant when controlling for age, education, ingroup/outgroup game orders, failing to understand the game, trait optimism, interpersonal trust, previous experience with a similar experiment, total contribution across both games, and ingroup favoritism in other dimensions (Supplementary Table S2 and Supplementary Information, Section 3). These results suggest that oxytocin motivates ingroup favoritism 
a

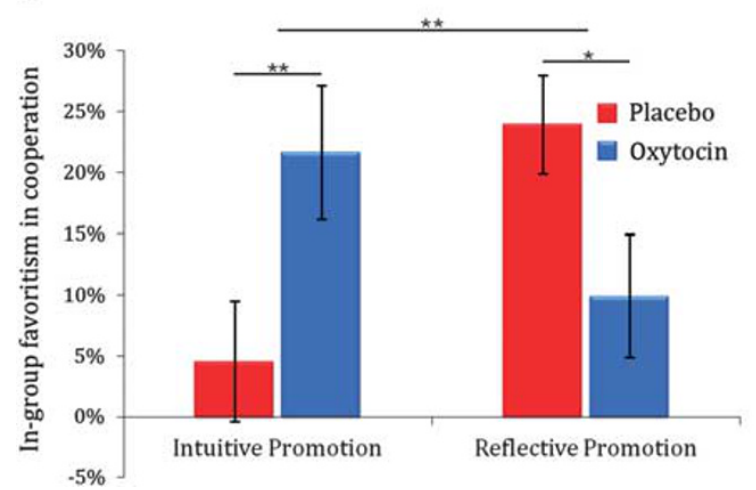

b

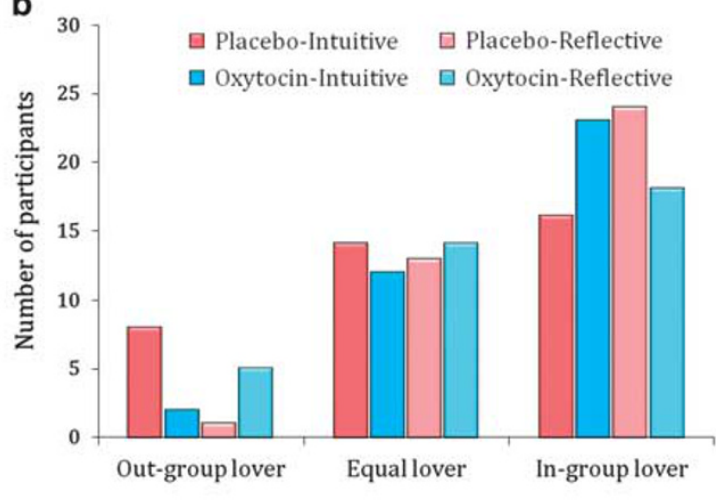

Figure I Distinct oxytocin effects on ingroup favoritism when intuition or reflection was promoted. (a) Oxytocin administration significantly enhanced ingroup favoritism when intuition was encouraged, whereas oxytocin significantly decreased ingroup favoritism when reflection was favored. (b) Distribution of outgroup-favored, equal, and ingroup-favored players. The distribution of 'ingroup-favored players' and 'outgroup-favored players' differed significantly across the four conditions. Oxytocin increased the number of ingroup-favored players among the individuals who were primed with intuition, whereas oxytocin decreased the number of ingroup-favored players among those who were primed with reflection.

a

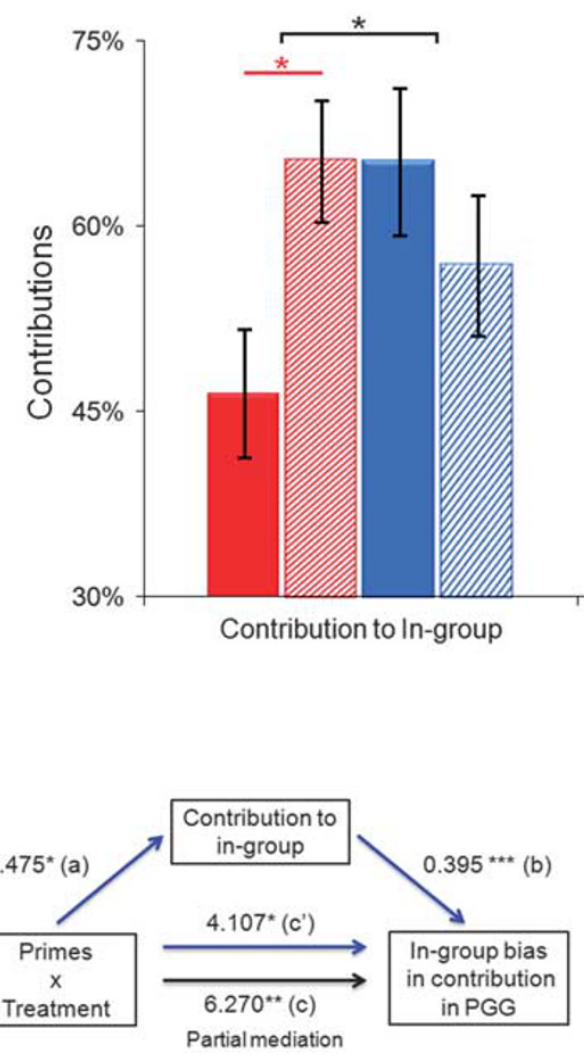

Placebo-Intuitive

OXT-Intuitive

Placebo-Reflective

OXT-Reflective
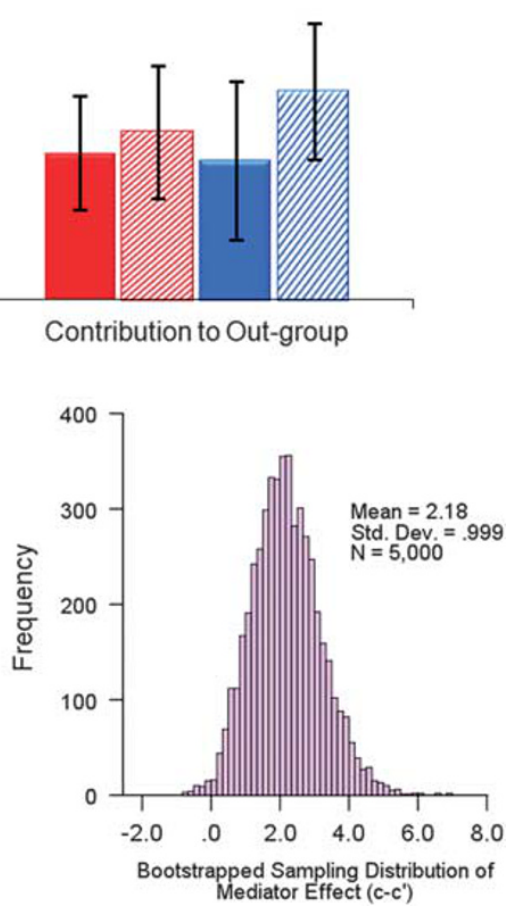

Figure 2 Effects on ingroup facilitation vs outgroup deterioration. (a) Oxytocin increased contribution to ingroup members when intuition was encouraged, but decreased ingroup cooperation when reflection was favored. However, there was no significant Treatment $\times$ Priming interaction when playing with outgroup members. (b) The Treatment $\times$ Priming interaction on ingroup favoritism was mediated by its effect on contribution amount to ingroup members. The bootstrapped sampling distribution of mediator effect was provided on the right panel.

through the intuitive system but inhibits ingroup favoritism when the reflective system is favored.

The Treatment $\times$ Priming interaction pattern was also evident in an analysis where we classified participants according to their differential contributions to ingroup and outgroup members. Among 150 participants, we identified 81 'ingroup-favored players' (who contributed more to ingroup compared with outgroup members) and 

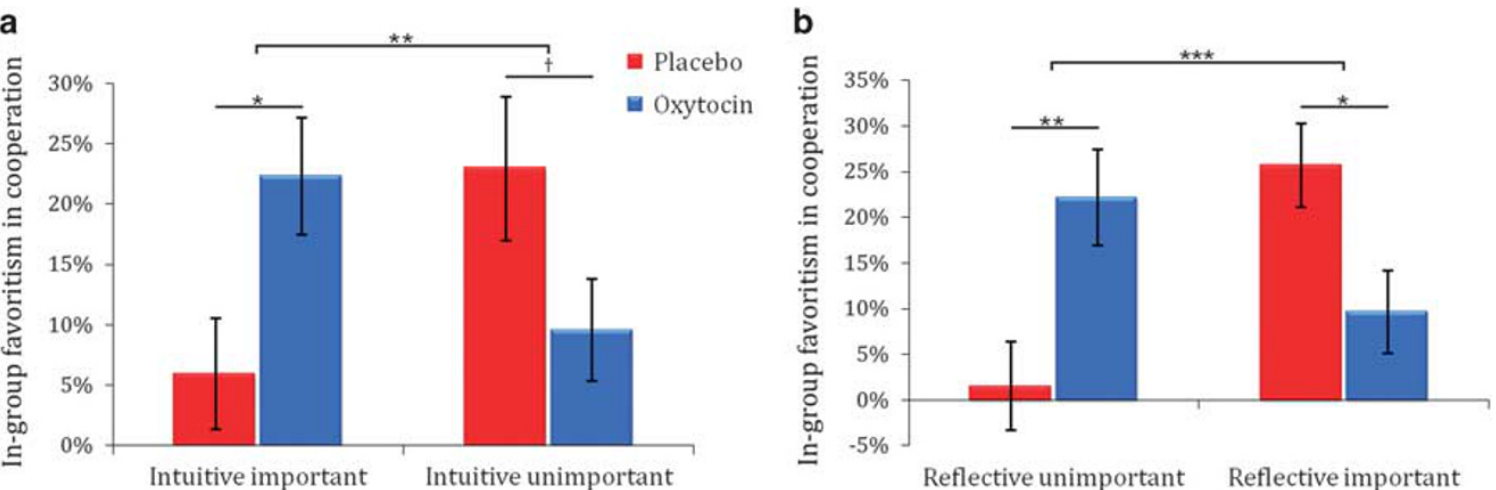

Figure 3 Influence of intuition vs reflection importance in daily life on ingroup favoritism during PGGs. Oxytocin administration increased ingroup favoritism on the contribution during PGG in individuals who thought intuition-important (a) or reflection-unimportant (b) in daily-life decision-making. However, oxytocin administration reduced ingroup favoritism during PGG in those who thought intuition-unimportant (a) or valued reflection-important (b) in daily-life decision-making. ${ }^{\dagger} P<0.07$; ${ }^{*} p<0.05$; ${ }^{*} *$ $p<0.01$; ${ }^{*} * * * 0.001$

16 'outgroup-favored players' (who contributed more to outgroup compared with ingroup members). The other 53 participants contributed equal amounts of money to ingroup and outgroup members. The distribution of 'ingroup-favored players' and 'outgroup-favored players' differed significantly across the four conditions $\left(\chi^{2}=9.543\right.$, Cramer's $V=0.314$ $(n=97), p=0.023)$. Cramer's $V$ coefficient suggested a moderate association between ingroup-/outgroup-favored player categorization and different conditions, that is, oxytocin increased the number of ingroup-favored players among the individuals who were primed with intuition (number of 'ingroup-favored players' $v s$ 'outgroup-favored players': placebo: $16 v s$ 8; oxytocin: $24 v s$ 1), whereas oxytocin decreased the number of ingroup-favored players among those who were primed with reflection (placebo: $23 v s$ 2; oxytocin: 18 vs 5; Figure 1b).

\section{Treatment $\times$ Priming Interaction on Contributions to Ingroup vs Outgroup Members}

To clarify whether the Treatment $\times$ Priming interaction on ingroup favoritism observed above was driven by cooperation with ingroup or outgroup members, we conducted separate analyses of contributions to ingroup and outgroup members. This analysis revealed a significant Treatment $x$ Priming interaction on contributions when playing PGG with ingroup members $(\mathrm{F}(1,146)=6.614, p=0.011$, $\eta^{2}=0.043$; Figure $2 \mathrm{a}$ and Supplementary Table S6) but not with outgroup members $(\mathrm{F}(1,146)=0.127, \quad p=0.722$, $\eta^{2}=0.001$; Supplementary Table S7). A mediation analysis further confirmed that the Treatment $\times$ Priming interaction on ingroup favoritism was mediated by its effect on contributions to ingroup members during PGG (Sobel test: $Z=-2.28, p=0.023$; Figure $2 b$, Supplementary Information, Section 4 and Supplementary Table S8) but not to outgroup members (Sobel test: $Z=-0.35, p=0.724$; Supplementary Table S9). A bootstrap resampling analysis of the effect size indicates that the mediator effects were different from zero with 95\% confidence (Figure 2b and Supplementary Information, Section 4 for details of bootstrap resampling analysis). These results indicated that the interaction between cognitive style and oxytocin treatment on ingroup favoritism arose mainly from the influences on participants' contributions towards ingroup members.

\section{Intuitive $v s$ Reflective Cognitive Styles in Daily Life}

We next tested how cognitive styles in daily decision-making affect oxytocin effect on ingroup-favoritism. Ingroupfavoritism on contributions during PGGs was subjected to 2 (Treatment: oxytocin vs placebo) $\times 2$ (Cognitive style: intuition-important $v s$ intuition-unimportant group or reflection-important $v s$ reflection-unimportant group) ANOVAs. These analyses revealed a similar pattern of results to those seen in our experimental manipulation by showing a significant interaction between Treatment and Cognitive style (Treatment $\times$ intuition importance: $F$ $(1,146)=8.863, p=0.003, \eta^{2}=0.057$ (Figure 3a); Treatment $\times$ reflection importance: $\mathrm{F}(1,146)=14.198, \quad p<0.001$, $\eta^{2}=0.089$ (Figure $\left.3 b\right)$ ). The Treatment $\times$ Cognitive style interaction on ingroup favoritism remained significant after controlling for age, education, ingroup/outgroup game orders, failing to understand the game, trait optimism, interpersonal trust, total contribution, and ingroup favoritism in other dimensions (Supplementary Tables S10-S13). Oxytocin ( $v s$ placebo) increased ingroup favoritism in the 'reflection-unimportant' group $(\mathrm{F}(1,71)=9.272, p=0.003$, $\left.\eta^{2}=0.116\right)$ and 'intuition-important' group $(\mathrm{F}(1,74)=5.714$, $p=0.019, \eta^{2}=0.072$ ), but decreased ingroup favoritism in the 'reflection-important' group $(\mathrm{F}(1,75)=5.365, p=0.023$, $\eta^{2}=0.067$ ) and 'intuition-unimportant' group (although this latter effect was only marginally significant; $F(1,72)=3.411$, $\left.p=0.069, \eta^{2}=0.045\right)$. These results provided additional evidence for the opposing oxytocin effects on ingroup favoritism in participants favoring intuitive $v s$ reflective systems.

\section{Prosociality $v s$ Expectations}

Finally, we tested whether the Treatment $\times$ Cognitive-style interaction on ingroup favoritism during the PGG resulted from differential expectation of contributions from ingroup and outgroup members. We asked participants to report their expectations of other players' contributions. Participants expected significantly more contributions from 
ingroup players compared with outgroup players $\left(\mathrm{F}(1,144)=81.867, p<0.001, \eta^{2}=0.362\right)$. However, neither the main effect of Treatment/Cognitive styles (either temporarily promoted or adapted in daily-life decisionmaking) nor their interaction was significant on expectations of differential contributions from ingroup compared with outgroup individuals ( $p$ s $>0.05$; Supplementary Figure S3). Thus, the ingroup favoritism in expectations did not vary across treatment and cognitive-style and the Treatment $x$ Cognitive-style interaction on ingroup favoritism cannot be simply caused by more optimistic expectations about the behaviors of ingroup members.

\section{DISCUSSION}

It has been documented that oxytocin motivates ingroup favoritism during social interaction (De Dreu, 2012; van IJzendoorn and Bakermans-Kranenburg, 2012). Here we revealed that oxytocin produced opposite effects on ingroup favoritism during social cooperation depending on individuals' cognitive styles. Specifically, intranasal administration of oxytocin ( $v s$ placebo) increased ingroup-favored cooperation among participants who were primed to be intuitive or preferred intuition in daily life but decreased ingroup favoritism in participants who were primed with reflection or preferred reflective decision-making in daily life. Our findings indicate that the biological and cognitive processes involved in social cooperation interact in a specific manner, that is, the adoption of intuition $v s$ reflection qualitatively changes the oxytocin effect on social cooperative behavior. The distinct oxytocin effects on ingroup favoritism are evident both when intuition and reflection are temporarily promoted by an experimental manipulation, and when intuition and reflection are preferred in daily life.

Oxytocin did not affect ingroup favoritism simply by changing decision speed because oxytocin ( $v s$ placebo) administration did not affect participants' decision times (see Supplementary Information, Section 5) and the interaction of oxytocin and cognitive style remained salient after controlling for decision times during PGG. In addition, although participants expected more contributions from ingroup members compared with outgroup members, this ingroup biased expectation was not altered by oxytocin or cognitive style or their interaction. Thus, oxytocin and cognitive style interactively affected participants' prosocial preferences rather than simply making them more or less optimistic about others' contributions. Moreover, we showed evidence that the distinct oxytocin effects on ingroup favoritism in intuitive and reflective minds cannot be explained by its effect on general risk attitudes in economic decisions (this was tested in a typical economic risk game in Experiment 2, see Supplementary Information, Section 6 for details), calculation ability (this was tested using a calculation task in Experiment 3, Supplementary Information, Section 7 for details), comprehension of the game rule, trust rating of the experiment, total contribution to ingroup and outgroup, and ingroup favoritism in first impression or likeness.

Oxytocin influences multiple processes involved in social cognition, such as enhancing social categorization of others, promoting social trust and empathy, and increasing ingroup favoritism (although the effect size was from weak to moderate; van IJzendoorn and Bakermans-Kranenburg, 2012). Our findings indicate that oxytocin administration increases ingroup-favored cooperation in intuitive minds but decreases ingroup favoritism in reflective minds in the same social situation. There are two ways to reconcile previous findings of general oxytocin effects in motivating ingroup favoritism with the current finding. First, it is likely that people rely on intuition in most cases, only turning to reflection when intuition fails, or when forced or habituated to reflect (Lieberman, 2007). Second, it is well recognized that emotional processing is linked to the intuitive rather than reflective system (Evans, 2008; Lieberman, 2007). The previous oxytocin effects on ingroup favoritism were mainly observed in the contexts of emotional situations, for example, ingroup favoritism in empathy for other's painful feeling (Sheng et al, 2013), ingroup-favored behaviors in an intergroup competition setting (De Dreu et al, 2010, 2011), or emotional moral judgment (De Dreu et al, 2011). Therefore, it is possible that the previous findings mainly reflect the oxytocin effect when intuition is favored (either by default or activated by emotional system).

The interaction between cognitive style and oxytocin was not driven by a ceiling effect of ingroup favoritism in reflective minds because oxytocin administration significantly decreased ingroup favoritism in reflective minds. Thus, it could be that, while oxytocin increases ingroup favoritism in intuitive minds, oxytocin may increase decisions to protect self-interest in reflective minds. Although oxytocin facilitates prosocial, approach, and parochial behaviors (Striepens et al, 2011), it also promotes risk aversion (Declerck et al, 2010) and envy (ShamayTsoory et al, 2009). Oxytocin enhances people's cooperative behaviors only when they are not overwhelmed by greed or self-interest. The oxytocin effect on facilitating cooperation was reduced when the greed or self-interest component is added into the economic games (Declerck et al, 2010). Because reflection can lead to more self-interest focused and less cooperative behaviors (Rand et al, 2012), it is possible that oxytocin administration increases the strategy to protect self-interest in a social situation when reflection leads to increased greed or selfishness.

Two findings regarding the effect of cognitive style on contributions during PGG (under placebo) were not anticipated. First, participants with intuitive minds did not show significant ingroup favoritism during cooperation under placebo (contribution to ingroup $v s$ outgroup members: $46.48 \pm 5.19 \%$ vs $41.91 \pm 4.64 \%, \quad \mathrm{~F}(1,37)=0.86$, $p=0.36)$. A possible way to account for this is that the group identity in the current work was built on trivial features and no explicit intergroup conflict was introduced. In such a condition, intuitive minds might fail to consider intragroup interests or intergroup conflict during cooperation. Future research should examine how the effects observed here are modulated by diverse group types (such as racial/ethnic groups or political parties) and/or situations (such as when facing intergroup competition/conflict or when outgroup threat is detected). Second, there was greater ingroup favoritism in reflective minds compared with intuitive minds under placebo (see Supplementary Information, Section 8 for detailed results and discussion). This result is perhaps surprising in light of previous findings that intuition promotes cooperation, whereas reflection 
increases selfish decisions (Rand et al, 2012). These results can be better understood when considering different meanings of a decision in a group or non-group situation. A person can act as either an individual self to pursue one's own goal/benefit or as a group self to pursue the goal/benefit of a social group (Leach et al, 2008; Ellemers, 2012). In a situation without group identity, reflection provides time to allow consideration of one's own benefit, leading to less contribution to the public pool (Rand et al, 2012). In a group situation, reflection provides time to ponder one's social group affiliation and the benefits of one's own group, leading to more contribution when playing with ingroup members (ie, stronger ingroup favoritism in the current work). There may also be an important cultural dimension to this finding - reflection may be more likely to lead to group affiliation among people from collectivist cultures (such as our participants), whereas those from individualistic cultures may favor selfishness when deliberating, regardless of group affiliation (Rand et al, 2015). These can be clarified in future research.

The current study was conducted on a sample of Chinese male adults. This raised the question whether and how the current effect can be generalized to other populations. Previous studies have shown differential or even opposing oxytocin effects between males and females (Macdonald, 2012; Fischer-Shofty et al, 2013; Rilling et al, 2014). Gender differences were also observed in ingroup favoritism (van Vugt et al, 2007; Charness and Rustichini, 2011). Another related issue is whether the current finding can be generalized to individuals from other cultures. The majority of literature on oxytocin effect has been conducted on non-Chinese (eg, European) populations. The current study of a Chinese population adds cultural diversity to the studied populations and raises an interesting question whether the effects of oxytocin on social cognition are sensitive to one's cultural background. On one hand, there were cultural differences in ingroup favoritism (Chen et al, 1998; Gelfand et al, 2012) and in oxytocin effect on affective responses to ostracism (Pfundmair et al, 2014). On the other hand, similar oxytocin effects on the promotion of ingroup favoritism were observed in European (De Dreu et al, 2010, 2011) and Chinese participants (Sheng et al, 2013). Moreover, similar to our finding, recent studies conducted on European participants showed that the oxytocin-driven group-serving dishonesty was relatively fast (Shalvi and De Dreu, 2014) and oxytocin reduced (deliberated) greedy decisions (De Dreu et al, 2015). It is important for future research to test directly whether and how the current findings can be generalized to females and other cultural populations.

It has been shown that the effects of oxytocin varied as a function of personal condition (such as psychopathology, personality trait, attachment style; Bartz et al, 2011a, b; Scheele et al, 2014). For example, the effect of oxytocin was discrepant in borderline personality disorder patients and healthy controls, as oxytocin decreased (rather than increased) trust and cooperation in patients (Bartz et al, 2011b). Moreover, oxytocin decreased trusting expectations for participants with anxious attachment but had no effect in less anxiously attached participants (Bartz et al, 2011b). Consistent with our findings in the context of social cooperation, a recent meta-analysis of facial recognition (Shahrestani et al, 2013) revealed distinct oxytocin effects in implicit ( $<300 \mathrm{~ms}$, fast, automatic processing) and explicit (>300 ms, slow, deliberated, controlled processing) contexts. Oxytocin enhances recognition of happy and angry expressions under implicit recognition (intuitive system) but facilitates recognition of fear expressions with longer durations of exposure (reflective system). These findings together indicate that intuitive $v s$ reflective processing can qualitatively change oxytocin effects on social cognition and emotional processing. Thus, individuals' personal milieu (such as cognitive styles, attachment styles) should be taken into consideration in future clinical research. Oxytocin is emerging as a pharmacological target for novel treatment in clinical trials with a wide variety of clinical groups (Heinrichs et al, 2009; Stavropoulos and Carver, 2013). However, patients from different clinical groups are characterized by different cognitive styles (eg, reflexive/intuitive $v s$ reflective/ rational). For example, there were high- and low-impulsive subtypes of social anxiety disorder (Kashdan and Hofmann, 2008; Binelli et al, 2014). Bipolar patients were more intuitive than unipolar depressed patients (Liebowitz et al, 1979; Janowsky et al, 1999). Autism patients were characterized by deficient intuition and lacked spontaneous adaptation (Allman et al, 2005; De Martino et al, 2008). A recent study has shown behavioral and psychophysiological evidence that autism patients were more rational and less likely to be guided by their intuition and emotional context in a financial task (De Martino et al, 2008). Our findings of opposing oxytocin effects on social cognition draw caution to the oxytocin pharmacotherapy for social dysfunction in that whether the effects of oxytocin on social functioning or emotional processing are facilitative, debilitative, or null, depending on an individual's cognitive style (Bartz et al, 2011a).

One of the most exciting areas of psychiatric neuroscience is to bridge the insights emerging from studies of social behavior in animals to human clinical research. However, the translational bridge needs to be built with careful consideration of species differences and unique human features. Our findings demonstrate that unique human cognitive processes (eg, reflective thinking) produce strong constraints on the function of oxytocin and can qualitatively change the effects of oxytocin on social behavior. This has critical implications in the translation of the oxytocin effect from animal models to clinical populations, and draws caution to map animal findings directly on to human neurobiology. In addition, our previous work has documented genetic modulation on pharmacological challenge (ie, serotonin transporter gene modulated the efficiency of serotonergic drugs; Ma, 2015; Ma et al, 2015); therefore, it is of critical interest for future research to further test how psychological and biological factors influence the effects of oxytocin.

\section{FUNDING AND DISCLOSURE}

The authors declare no conflict of interest.

\section{ACKNOWLEDGMENTS}

We thank Chenbo Wang, Bingfeng Li, Qiaodan Luo and Guofeng Ma for research assistance. We thank Caroline Zink for very insightful discussion on an early draft. This work 
was supported by the National Natural Science Foundation of China (Project Nos 31421003, 31470986, 91332125) and the Ministry of Education of China (Project No. 20130001110049), the National Institute of Mental Health (R01MH059282) and the John Templeton Foundation.

\section{REFERENCES}

Allman JM, Watson KK, Tetreault NA, Hakeem AY (2005). Intuition and autism: a possible role for Von Economo neurons. Trends Cogn Sci 9: 367-373.

Arrow H (2007). Evolution. The sharp end of altruism. Science 318: 581-582.

Bakermans-Kranenburg MJ, van IJzendoorn MH (2013). Sniffing around oxytocin: review and meta-analyses of trials in healthy and clinical groups with implications for pharmacotherapy. Transl Psychiatry 3: e258.

Bartz JA, Hollander E (2006). The neuroscience of affiliation: forging links between basic and clinical research on neuropeptides and social behavior. Horm Behav 50: 518-528.

Baumgartner T, Heinrichs M, Vonlanthen A, Fischbacher U, Fehr E (2008). Oxytocin shapes the neural circuitry of trust and trust adaptation in humans. Neuron 22: 639-650.

Bartz JA, Zaki J, Bllander E, Ludwig NN, Kolevzon A, Ochsner KN (2010). Oxytocin selectively improves empathic accuracy. Psychol Sci 21: 1426-1428.

Bartz JA, Zaki J, Bolger N, Ochsner KN (2011a). Social effects of oxytocin in humans: context and person matter. Trends Cogn Sci 15: 301-309.

Bartz JA, Simeon D, Hamilton H, Kim S, Crystal S, Braun A, Vicens V, Hollander E (2011b). Oxytocin can hinder trust and cooperation in borderline personality disorder. Soc Cogn Affect Neurosci 6: 556-563.

Binelli C, Muñiz A, Sanches S, Ortiz A, Navines R, Egmond E et al (2014). New evidence of heterogeneity in social anxiety disorder: defining two qualitatively different personality profiles taking into account clinical environmental and genetic factors. Eur Psychiatry 30: $160-165$.

Charness G, Rustichini A (2011). Gender differences in cooperation with group membership. Games Econ Behav 72: 77-85.

Chen YR, Brockner J, Katz T (1998). Toward an explanation of cultural differences in in-group favoritism: the role of individual versus collective primacy. J Pers Soc Psychol 75: 1490-1502.

Dalgleish T (2004). The emotional brain. Nat Rev 5: 582-589.

De Dreu CKW (2012). Oxytocin modulates cooperation within and competition between groups: an integrative review and research agenda. Horm Behav 61: 419-428.

De Dreu CKW, Greer LL, van Kleef GA, Shalvi S, Handgraaf MJ (2011). Oxytocin promotes human ethnocentrism. Proc Natl Acad Sci USA 108: 1262-1266.

De Dreu CKW, Scholte HS, van Winden FA, Ridderinkhof KR (2015). Oxytocin tempers calculated greed but not impulsive defense in predator-prey contests. Soc Cogn Affect Neurosci (doi:10.1093/scan/nsu109).

De Dreu CKW, Greer LL, Handgraaf MJ, Shalvi S, van Kleef GA, Baas $M$ et al (2010). The neuropeptide oxytocin regulates parochial altruism in intergroup conflict among humans. Science 328: $1408-1411$.

De Martino B, Harrison NA, Knafo S, Bird G, Dolan RJ (2008). Explaining enhanced logical consistency during decision making in autism. J Neurosci 28: 10746-10750.

Declerck CH, Boone C, Kiyonari T (2010). Oxytocin and cooperation under conditions of uncertainty: the modulating role of incentives and social information. Horm Behav 57: 368-374.

Domes G, Heinrichs $M$, Gläscher J, Büchel C, Braus DF, Herpertz SC (2007). Oxytocin attenuates amygdala responses to emotional faces regardless of valence. Biol Psychiatry 62: 1187-1190.
Donaldson ZR, Young LJ (2008). Oxytocin vasopressin and the neurogenetics of sociality. Science 322: 900-903.

Ellemers N (2012). The group self. Science 336: 848-852.

Evans JS (2008). Dual-processing accounts of reasoning judgment and social cognition. Annu Rev Psychol 59: 255-278.

Fischer-Shofty M, Levkovitz Y, Shamay-Tsoory SG (2013). Oxytocin facilitates accurate perception of competition in men and kinship in women. Soc Cogn Affect Neurosci 8: 313-317.

Gelfand M, Shteynberg G, Lee T, Lun J, Lyons S, Bell C et al (2012). The cultural contagion of conflict. Philos Trans $R$ Soc Ser B 367: 692-703.

Gordon I, vander Wyk BC, Bennett RH, Cordeaux C, Lucas MV, Eilbott JA et al (2013). Oxytocin enhances brain function in children with autism. Proc Natl Acad Sci USA 110: 20953-20958.

Groppe SE, Gossen A, Rademacher L, Hahn A, Westphal L, Gründer $\mathrm{G}$ et al (2013). Oxytocin influences processing of socially relevant cues in the ventral tegmental area of the human brain. Biol Psychiatry 74: 172-179.

Heinrichs M, von Dawans B, Domes G (2009). Oxytocin vasopressin and human social behavior. Front Neuroendocrinol 30: $548-557$.

Insel TR (2010). The challenge of translation in social neuroscience: a review of oxytocin vasopressin and affiliative behavior. Neuron 65: 768-779.

Janowsky DS, Morter S, Hong L, Howe L (1999). Myers Briggs Type Indicator and Tridimensional Personality Questionnaire differences between bipolar patients and unipolar depressed patients. Bipolar Disord 1: 98-108.

Kahneman D (2011). Thinking Fast and Slow. Straus and Giroux.

Kashdan TB, Hofmann SG (2008). The high-novelty-seeking impulsive subtype of generalized social anxiety disorder. Depress Anxiety 25: 535-541.

Kosfeld M, Heinrichs M, Zak PJ, Fischbacher U, Fehr E (2005). Oxytocin increases trust in humans. Nature 435: 673-676.

Leach CW, van Zomeren M, Zebel S, Vliek ML, Pennekamp SF, Doosje B et al (2008). Group-level self-definition and selfinvestment: a hierarchical (multicomponent) model of ingroup identification. J Pers Soc Psychol 95: 144-165.

Lieberman MD (2007). Social cognitive neuroscience: a review of core processes Annu Rev Psychol 58: 259-289.

Liebowitz MR, Stallone F, Dunner DL, Fieve RF (1979). Personality features of patients with primary affective disorder. Acta Psychiatr Scand 60: 214-224.

Ludwig M, Leng G (2006). Dendritic peptide release and peptidedependent behaviors. Nat Rev Neurosci 7: 126-136.

Ma Y, Li B, Zhang W, Rao Y, Han S (2015). Allelic variation in 5-HTTLPR and the effects of citalopram on the emotional neural network. Br J Psychiatry; e-pub ahead of print 5 March 2015. (doi:10.1192/bjp.bp.114.150128).

Ma Y (2015). Neuropsychological mechanism underlying antidepressant effect: a systematic meta-analysis. Mol Psychiatry 20: 311-319.

Macdonald KS (2012). Sex receptors and attachment: a review of individual factors influencing response to oxytocin. Front Neurosci 6: 194.

Meyer-Lindenberg A, Domes G, Kirsch P, Heinrichs M (2011). Oxytocin and vasopressin in the human brain: social neuropeptides for translational medicine. Nat Rev Neurosci 12: 524-538.

Miller EK, Cohen JD (2001). An integrative theory of prefrontal cortex function. Annu Rev Neurosci 24: 167-202.

Nowak MA, Sigmund K (2005). Evolution of indirect reciprocity. Nature 437: 1291-1298.

Petrovic P, Kalisch R, Singer T, Dolan RJ (2008). Oxytocin attenuates affective evaluations of conditioned faces and amygdala activity. J Neurosci 28: 6607-6615.

Pfundmaira M, Aydinc N, Freya D, Echterhoff G (2014). The interplay of oxytocin and collectivistic orientation shields against negative effects of ostracism. J Exp Soc Psychol 55: 246-251. 
Rand DG, Greene JD, Nowak MA (2012). Spontaneous giving and calculated greed. Nature 489: 427-430.

Rand DR, Newman GE, Wurzbacher OM (2015). Social context and the dynamics of cooperative choice. J Behav Dec Making 28: $159-166$

Rand DG, Nowak MA (2013). Human cooperation. Trends Cogn Sci 17: 413-425.

Rilling JK, Demarco AC, Hackett PD, Chen X, Gautam P, Stair S et al (2014). Sex differences in the neural and behavioral response to intranasal oxytocin and vasopressin during human social interaction. Psychoneuroendocrinology 39: 237-248.

Scheele D, Kendrick KM, Khouri C, Kretzer E, Schläpfer TE, Stoffel-Wagner B et al (2014). An oxytocin-induced facilitation of neural and emotional responses to social touch correlates inversely with autism traits. Neuropsychopharmacology 39: 2078-2085.

Shahrestani S, Kemp AH, Guastella AJ (2013). The impact of a single administration of intranasal oxytocin on the recognition of basic emotions in humans: a meta-analysis. Neuropsychopharmacology 38: 1929-1936.

Shalvi S, De Dreu CKW (2014). Oxytocin promotes group-serving dishonesty. Proc Natl Acad Sci USA 111: 5503-5507.

Shamay-Tsoory SG, Fischer M, Dvash J, Harari H, Perach-Bloom N, Levkovitz Y (2009). Intranasal administration of oxytocin increases envy and schadenfreude (gloating). Biol Psychiatry 66: 864-870.

Sheng F, Liu Y, Zhou B, Zhou W, Han S (2013). Oxytocin modulates the racial bias in neural responses to others' suffering. Biol Psychol 92: 380-386.

Stavropoulos KK, Carver LJ (2013). Research review: social motivation and oxytocin in autism-implications for joint attention development and intervention. J Child Psychol Psychiatry 54: 603-618.

Striepens N, Kendrick KM, Maier W, Hurlemann R (2011). Prosocial effects of oxytocin and clinical evidence for its therapeutic potential. Front Neuroendocrinol 32: 426-450.

Tajfel H, Billig MG, Bundy RP, Flament C (1971). Social categorization and intergroup behavior. Eur J Soc Psychol 1: 149-178.

van IJzendoorn MH, Bakermans-Kranenburg MJ (2012). A sniff of trust: meta-analysis of the effects of intranasal oxytocin administration on face recognition trust to ingroup and trust to outgroup. Psychoneuroendocrinology 37: 438-443.

van Vugt M, de Cremer D, Janssen DP (2007). Gender differences in cooperation and competition: the male-warrior hypothesis. Psychol Sci 18: 19-23.

Zaki J, Mitchell JP (2013). Intuitive prosociality. Curr Dir Psychol Sci 22: $466-470$.

Supplementary Information accompanies the paper on the Neuropsychopharmacology website (http://www.nature.com/npp) 